\title{
Magali Nachtergael et Lucille Toth (dir.), Danse contemporaine et littérature. Entre fictions et performances écrites
}

\section{Francesca Forcolin}

\section{(2) OpenEdition \\ Journals}

Edizione digitale

URL: http://journals.openedition.org/studifrancesi/1516

DOI: ERREUR PDO dans/localdata/www-bin/Core/Core/Db/Db.class.php L.34 : SQLSTATE[HY000]

[2006] MySQL server has gone away

ISSN: 2421-5856

\section{Editore}

Rosenberg \& Sellier

\section{Edizione cartacea}

Data di pubblicazione: 1 décembre 2015

Paginazione: 654-655

ISSN: 0039-2944

Notizia bibliografica digitale

Francesca Forcolin, «Magali Nachtergael et Lucille Toth (dir.), Danse contemporaine et littérature. Entre fictions et performances écrites», Studi Francesi [Online], 177 (LIX | III) | 2015, online dal 01 décembre 2015, consultato il 08 janvier 2021. URL: http://journals.openedition.org/studifrancesi/1516 ; DOI: https://doi.org/10.4000/studifrancesi.1516

Questo documento è stato generato automaticamente il 8 janvier 2021.

\section{(†)

Studi Francesi è distribuita con Licenza Creative Commons Attribuzione - Non commerciale - Non opere derivate 4.0 Internazionale. 


\title{
Magali Nachtergael et Lucille Toth (dir.), Danse contemporaine et littérature. Entre fictions et performances écrites
}

\author{
Francesca Forcolin
}

\section{NOTIZIA}

MAGALI NACHTERGAEL et LUCILLE TOTH (dir.), Danse contemporaine et littérature. Entre fictions et performances écrites, Centre National de la danse, Pantin, 2015, pp. 237.

1 Questo è un volume collettivo che parla di un passo a due: quello tra la letteratura e la danza, l'incontro/scontro tra due forme d'arte diverse, opposte, che sembrerebbero estranee l'una dall'altra, due sistemi semiotici divergenti come il corpo e lo spirito ma che si cercano, si scoprono, si toccano. È un legame che affiora all'inizio del secolo scorso con la nascita della danza moderna, quando, ad esempio, Isadora Duncan afferma di danzare seguendo la musicalità di alcuni testi poetici, o quando Martha Graham incorpora nei suoi balletti la scrittura di Emily Dickinson. Oggi, un gran numero di coreografie si appoggia a testi di diversa natura (filosofici, romanzeschi), reinterpretando, attraverso il gesto, la scrittura. Nel volume di Magali NACHTERGAEL et Lucille тотн (Introduction, pp. 7-15), sono raccolti studi di specialisti di varie discipline che spaziano tra le differenti culture, con l'obiettivo di sottoporre al lettore un panorama eterogeneo e variegato, in un'opera che si dichiara essere la prima a concentrarsi esclusivamente sul rapporto tra danza e letteratura. È suddivisa in quattro sezioni: la prima, «La littérature dansée», si concentra sulla rappresentazione coreografica di alcune opere letterarie, proponendo prima una sorta di quadro generale e poi entrando via via nello specifico con l'analisi di singoli adattamenti (Mise en corps, mise en voix: le texte et ses apparitions sur la scène choréographique contemporaine di Lucille 
Tотн, pp.17-22; Pourquoi la littérature mobilise-t-elle le corps dansant?, Alice GODFROY, pp. 23-30; La littérature sur un plateau: Dominique Bagouet à l'écoute d'Emmanuel Bove, Cécile SCHENCK, pp. 31-41; La poésie dans l'art chorégraphique contemporain, Biliana VASSILEVA, pp. 42-50; Faustin Linyekula: dynamiques postcoloniales de l'adaptation. "La Création du monde (1923-2012)" et "Pour en finir avec Bérénice", Carole MAccotTA, pp. 51-62).

2 Il secondo capitolo, «Écrivains en scène», si concentra sul Festival Concordan(s)e (Le festival Concordan(s)e: jeux d'influences entre texte et danse di Judith MAYER, pp. 83-87), giunto questa primavera alla sua nona edizione, e che si presenta come «une aventure singulière où un écrivain rencontre un chorégraphe»: è rivelatore di un lavoro complesso, in cui lo scrittore collabora con il coreografo entrando in scena ed esprimendosi con le parole, con i gesti, con la musica, come nel caso di Célia Houdart (Entretien avec Célia Houdart di Judith MAYER, pp. 88-93) o Olivia Rosenthal (Le pas de deux d'Olivia Rosenthal di Nancy MURzILLI, pp.94-100) che ha riconosciuto nel lavoro della coreografa Carlotta Sagna il tema comune della follia, espresso nell'opera a quattro mani Petite pièce avec Olivia.

3 Il terzo capitolo, «L'auto-: s'écrire sur et hors scène», esplora la presa di parola del danzatore/coreografo sul web, sui social, perché, pur non essendo nuova la pratica della scrittura in coloro che praticano la danza, nell'ultimo decennio questa ha preso piede in maniera significativa divenendo un'espressione di sé molto diffusa (Coming Out di Enora RIVIÈRE, pp. 127-134; L'autobiochorégraphie di Laura SOUDY, pp. 135-140; Blogs de danseurs: enjeux croisés di Alice GERVAIS-RAGU, pp. 141-146; Narration en mouvement: I AM 1984 de Barbara Matijevic et Giuseppe Chico di Katja SIMUNIC, pp. 147-151; «An account of an ongoing personal discourse»: la portée kinésique de l'écriture chez Simone Forti di Marie CANET, pp. 157-161).

4 La quarta sezione, "Écrire le mouvement: au-delà de la littérature», si sofferma sull'avant-texte, sugli schizzi, bozze, appunti, su tutti quei segni che esprimono il processo della creazione coreografica, testimoniando che il lavoro di scrittura è anche alla base della danza (Danser-Écrire: sur l'écriture du mouvement di Raphael cotTin, pp.183-190; Jan Fabre et Anne Teresa De Keersmaeker: asymétries du document di Magali NACHTERGAEL, pp. 191-197; Du théâtre à la danse: Roberto Zucco par Christian et François Ben Aïm di Lenaig LE YEUC'H, pp. 198-205).

5 Alle prime tre sezioni si aggiungono tre Carnets monografici che ampliano con ricerche e interviste l'intero volume, mettendo l'accento su alcuni degli incontri più significativi tra danza e scrittura: il primo analizza il lavoro di Maguy Marin (Carnet Maguy Marin, pp.63-81), che si è ispirata in particolar modo alla scrittura di Beckett mettendo in scena May B a partire da Fin de Partie e En attendant Godot. Troviamo successivamente l'intervento di Stefano GENETTI su Pascal Quignard (Carnet Pascal Quignard, pp. 103-125), che oltre a riportare la pratica scritturale dell'autore restituisce la sua parola diretta grazie a una approfondita intervista. L'ultimo Carnet è dedicato alla coreografa Mathilde Monnier (pp. 163-181), la quale concepisce il proprio lavoro dall'incontro con altri artisti: dalla collaborazione, ad esempio, con Christine Angot sono nati spettacoli che si presentano come un dialogo tra donne che condividono il fascino e il rigetto per l'ambiente sociale in cui sono nate e cresciute.

6 L'ultimo capitolo (Un livre: un chorégraphe, pp. 207-214) riunisce brevi dialoghi con alcuni coreografi (Chouinard, Gérard, Grandville, Rizzo) che delineano l'importanza della scrittura per il proprio lavoro. Chiude la Post-face, le danseur meurtri (extraits) di 
Daniel DoBbels (pp. 215-222), a suggello di un interessantissimo volume collettivo, di piacevole lettura e ricco di spunti. 